\title{
Development of Methods for the Preparation of Process Solutions for the Determination of Natural Radionuclides and Tritium
}

\author{
Lydia G. Bondarevaa,b* and Ivan G. Tananaev \\ ${ }^{a}$ Siberian Federal University \\ 79 Svobodny, Krasnoyarsk, 660041, Russia \\ ${ }^{b}$ Igarka's Permafrost Laboratory of the Permafrost Institute SB RAS \\ 8 1st md., Igarka, 663200, Russia \\ ${ }^{c}$ Ozerskii Institute of Technology Branch of the \\ National Research Nuclear University \\ Moscow Institute of Physics and engineer \\ 48 Pobedy, Ozersk, 456783, Russia
}

Received 02.11.2014, received in revised form 12.12.2014, accepted 20.01.2015

\begin{abstract}
Drilling water of the Siberian oil deposits and that from the ground waters of Yakutia are the objects of study. Tritium has been determined by liquid scintillation spectrometry with inductively coupled plasma and the element content - by mass spectrometry. Special equipment has been developed which allows managing little or no change in the physical properties of the fluids and separating the main salt matrix (inorganic salts, trace of oil and polymers) of the determined submicro elements and radionuclides (uranium, thorium and tritium isotopes). The development of the equipment has significantly improved the detection limit of uranium (20 $\mathrm{ng} / \mathrm{L})$, thorium $(10 \mathrm{ng} / \mathrm{L})$ and tritium $(1 \mathrm{~Bq} / \mathrm{L})$, with the efficiency being $27 \%$.
\end{abstract}

Keywords: metals, radioactivity, associated brines, uranium, thorium, tritium.

(C) Siberian Federal University. All rights reserved

* Corresponding author E-mail address: lydiabondareva@gmail.com 


\title{
Разработка методики
}

\section{для подготовки технологических растворов \\ для определения}

\section{естественных радионуклидов и трития}

\author{
Л.Г. Бондарева ${ }^{\mathrm{a}, \tilde{\sigma} *}$, И.Г. Тананаев \\ ${ }^{a}$ Сибирский федеральный университет \\ Россия, 660041, Красноярск, пр. Свободный, 79 \\ ${ }^{6}$ Игарская геокриологическая лаборатория \\ Института мерзлотоведения СО РАН \\ Россия, 663200, Игарка, 1-й микрорайон, 8 \\ ${ }^{6}$ Озерский технологический институт \\ филиал Национального исследовательского \\ ядерного университета МИФИ \\ Россия, 456783, Озерск, пр. Победы, 48
}

В данной статье приводятся результаты исследования влияния трития и естественных радионуклидов буровых вод в местах разработки сибирских нефтяных месторождений и грунтовых водах Якутии. Работы проводили с использованием принципиально новой методики. Традиционный метод определения трития предполагает жидкостную сцинтилляционную спектрометрию, содержание элементов - масс-спектрометрию с индуктивно связанной плазмой. Было разработано специальное оборудование, которое позволило выделять жидкости, содержащие искомые элементы в субмикроколичествах (урана, тория и изотопы трития) практически без изменений в физическо-химических свойствах из сложных солевых матрии (неорганические соли, следы нефти и полимеров). Предложенная методика значительно

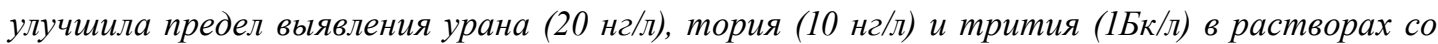
сложной матрищей, с эффективностью измерения трития $27 \%$.

Ключевые слова: металль, радиоактивность, буровые рассолы, уран, торий, тритий.

В настоящее время исследования показывают, что разработка месторождений горючих полезных ископаемых с каждым годом приводит к все большим нагрузкам на природную среду. При этом существует многообразие негативных процессов, которые ведут к деградации почв, ухудшению почвенного плодородия, нарушению экологии почв, ландшафтов и другим негативным факторам. До сих пор считалось, что наиболее опасными последствиями для природной среды выступали содержащиеся в составе нефтяных отходов углеводороды, однако в последние десятилетия в составе нефти, газа и пластовых вод были обнаружены десятки различных металлов, галогенов и радиоактивных элементов. Таким образом, одной из основных причин техногенной нагрузки на почвы, как и всей наземной экосистемы, является загрязнение различными токсикантами, в том числе тяжелыми металлами и радиоактивными элементами $[1,2]$. 
Как известно, уран и торий относятся к группе токсичных элементов [3]. Это вызывает необходимость строгого контроля их поступления в окружающую среду, что требует на практике использования сравнительно недорогих, доступных, экспрессных и высокочувствительных методов определения этих элементов. Для снижения предела обнаружения микроколичеств элементов любым методом требуется предварительное концентрирование ионов металлов из больших объемов воды. С этой целью еще часто используют выпаривание больших объемов проб, осаждение, соосаждение и т.д. [4]. При этом происходит концентрирование не только определяемых микрокомпонентов, но и матричных элементов, что снижает точность и воспроизводимость результатов. Прямое определение элементов физическими и физикохимическими методами также не всегда возможно из-за значительного влияния матричного состава пробы. Таким образом, применяемые традиционные методы анализа в ряде случаев не удовлетворяют современным требованиям по экспрессности, точности и чувствительности обнаружения следовых количеств элементов-токсикантов, что стало поводом для поиска и разработки новых способов их выделения и концентрирования.

Кроме того, выбор метода для анализа проб окружающей среды, особенно их пробоподготовки к измерению, должен проводиться с учетом требований пользователя и/или требований правовых актов, регулирующих содержание естественных радионуклидов. Основной задачей для решения такого вопроса служит выбор наиболее подходящих и соответствующих методов для анализа $[5,6]$. Метод определения должен учитывать цель анализа; тип образца, в том числе матрицы образца; какие типы изотопов будут определены; предполагаемую концентрацию конкретных природных изотопов и ряд других параметров.

Следует отметить, что при анализе жидкостей со сложной матрицей, какой являются буровые растворы, а также грунтовые воды, особую сложность представляет определение на фоне больших концентраций макроэлементов, таких как $\mathrm{Na}, \mathrm{Ca}, \mathrm{K}, \mathrm{Mg}$, и других элементов, находящихся в субмикроколичествах, в том числе и радионуклидов различного происхождения.

Целью приведенной работы стала разработка методики для подготовки жидких проб со сложной матрицей для определения естественных радионуклидов (уран и торий) и трития.

\section{Экспериментальная часть}

Учитывая, что буровые растворы являются наиболее токсичной частью буровых отходов, в качестве объектов исследований были использованы их пробы, отобранные на одном из нефтяных месторождений Сибири. Физико-химический состав исследуемых буровых вод представлен в табл. 1 .

В работе также использовали подземные воды (n=6) со значительным содержанием минеральных солей, отобранных в Республике Саха (Якутия). В последнем случае это были бессульфатные Cl-Na-Ca рассолы с минерализацией более 100 г/л, излившиеся из буровых скважин.

Часть исходных растворов ( $\mathrm{n}=6$, по 3 из каждого района исследования) подвергалась традиционной подготовке для последующего определения содержания урана и тория методом альфа-спектрометрии. Результаты были необходимы для последующего сравнения содержаний урана и тория, полученных в ходе применения разрабатываемой методики.

Уран и торий в образцах выделяли с помощью смолы Dowex 1х2 и UTEVA (EichromTechnologies). ${ }^{232} \mathrm{U}$ и ${ }^{229} \mathrm{Th}$ были взяты в качестве радиоактивной метки [6-8]. Количе- 
Таблица 1. Физико-химический состав исследуемых буровых сточных вод (n=6)

\begin{tabular}{|c|c|c|}
\hline Наименование показателей & Единица измерения & Значения \\
\hline $\mathrm{pH}$ & $\sigma / p$ & $7-10$ \\
\hline Плотность & $\Gamma / \mathrm{cm}^{3}$ & $1,0-1,2$ \\
\hline Механические примеси & мг/л & $1800-3300$ \\
\hline Сухой остаток & мГ/л & $2800-6030$ \\
\hline Общая минерализация & мГ/л & $1300-2600$ \\
\hline
\end{tabular}

ственное определение изотопов урана и тория проводили методом альфа-спектрометрии, эффективность детектора которого определяли сертифицированным альфа-излучающим источником из Analytics Inc (Модель SRS 63997-121).

В работе использовали альфа-спектрометр - Alpha Analyst (Canberra), (ИГМ СО РАН, Новосибирск). Для определения трития применяли ультранизкофоновый жидкостносцинтилляционный альфа-бета-спектрометр Quantulus1220 (Perkin Elmer, США) (Центр коллективного пользования Красноярского научного центра СО РАН). Масс-спектрометр ICP-MS (масс-спектрометр с индуктивно связанной плазмой) X Series 2, ThermoScientific корпорации (США) был задействован для определения микроэлементов в начальных и отделенных раствоpax (Центр коллективного пользования Красноярского научного центра СО РАН).

Основные источники погрешности исследований в определении урана и тория методом альфа-спектрометрии: погрешности измерения объема такие, как при работе с пипетками или приготовлении растворов определенного объема, неполное выделение урана и тория (химический выход $~ 54$ \%), возможные различия в составе между стандартными и анализируемыми и образцами.

Предел обнаружения для радиометрических методов устанавливается как минимально детектируемая активность (МДА), существует большое число подходов и алгоритмов определения [9-16].

Расчеты при выявлении предела обнаружения трития проводили по международным стандартным методам [17, 18].

Для пересчета полученной активности в массовые величины использовали следующую формулу:

$$
\mathrm{m}=\mathrm{A} \cdot \mathrm{A}_{\mathrm{r}} \cdot \mathrm{T}_{1 / 2} / \mathrm{N} \cdot \mathrm{N}_{\mathrm{A}},
$$

где $\mathrm{A}$ - активность (распад/с, Бк); $\mathrm{N}$ - количество радиоактивных атомов; $\mathrm{T}_{1 / 2}$ - период полураспада, c; $\mathrm{A}_{\mathrm{r}}$ - атомный вес (г/моль); $\mathrm{m}$ - масса, г; $\mathrm{N}_{\mathrm{A}}$ - число Авогадро = 6,022·10

Все расчеты, изложенные в статье, проводили с использованием статистического математического аппарата [20].

\section{Результаты и обсуждение}

Для того чтобы провести определение элементов, в исходных растворах из части каждой пробы была декантирована жидкость методом фильтрования, в которой затем проводили 
Таблица 2. Результаты содержания элементов в исходных пробах методом ИСП МС, мгк/л

\begin{tabular}{|c|c|c|c|c|c|c|c|}
\hline \multirow[b]{2}{*}{ № } & \multirow{2}{*}{ 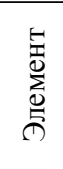 } & \multicolumn{2}{|c|}{ Объект исследования } & \multirow[b]{2}{*}{ № } & \multirow{2}{*}{ 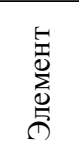 } & \multicolumn{2}{|c|}{ Объект исследования } \\
\hline & & $\begin{array}{c}\text { Район добычи } \\
\text { нефти, Сибирь } \\
(\mathrm{n}=6)\end{array}$ & $\begin{array}{c}\text { Подземные } \\
\text { рассолы, Якутия } \\
(\mathrm{n}=6)\end{array}$ & & & $\begin{array}{c}\text { Район добычи } \\
\text { нефти, Сибирь } \\
(\mathrm{n}=6)\end{array}$ & $\begin{array}{c}\text { Подземные } \\
\text { рассолы, Якутия } \\
(\mathrm{n}=6)\end{array}$ \\
\hline 1 & $\mathrm{Li}$ & $5,7(4,4-6,2)$ & $6,6(6,0-11)$ & 14 & $\mathrm{Ni}$ & $6,1(5,3-7,1)$ & $8(7,9-8,1)$ \\
\hline 2 & $\mathrm{~B}$ & $90(84-100)$ & $98(29-120)$ & 15 & $\mathrm{Cu}$ & $38(18-44)$ & $11(10-12)$ \\
\hline 3 & $\mathrm{C}$ & $\begin{array}{c}1600000 \\
(1000000- \\
23000000)\end{array}$ & $\begin{array}{c}680000 \\
(580000-720000)\end{array}$ & 16 & $\mathrm{Zn}$ & $8(7-9)$ & $31(8-81)$ \\
\hline 4 & $\mathrm{Na}$ & $\begin{array}{c}91000 \\
(89000-95000)\end{array}$ & $\begin{array}{c}710000 \\
(680000-740000)\end{array}$ & 17 & $\mathrm{Ga}$ & $3,1(2,1-3,6)$ & $4,8(3,0-5,2)$ \\
\hline 5 & $\mathrm{Mg}$ & $\begin{array}{c}8000 \\
(6000-10000)\end{array}$ & $\begin{array}{c}15000 \\
(8400-19000)\end{array}$ & 18 & As & $3,8(3,7-3,9)$ & $4,6(1,7-6,4)$ \\
\hline 6 & $\mathrm{Al}$ & $\begin{array}{c}340 \\
(330-350)\end{array}$ & $410(350-610)$ & 19 & $\mathrm{Se}$ & $710(700-720)$ & $730(720-740)$ \\
\hline 7 & $\mathrm{Si}$ & $\begin{array}{c}5200 \\
(4900-5900)\end{array}$ & $5000(4800-6000)$ & 20 & $\mathrm{Br}$ & $68(65-71)$ & $91(83-96)$ \\
\hline 8 & $\mathrm{P}$ & $\begin{array}{c}80 \\
(58-140)\end{array}$ & $100(48-200)$ & 21 & $\mathrm{Sr}$ & $420(230-500)$ & $510(380-810)$ \\
\hline 9 & $\mathrm{~S}$ & $\begin{array}{c}5800 \\
(4800-17000)\end{array}$ & $700(540-1200)$ & 22 & Mo & $3(2-4)$ & $5(4-7)$ \\
\hline 10 & $\mathrm{Cl}$ & $\begin{array}{c}5400 \\
(5300-5700)\end{array}$ & $6700(6600-6800)$ & 23 & $\mathrm{Cd}$ & $6(5-8)$ & $3(2-5)$ \\
\hline 11 & K & $\begin{array}{c}610 \\
(540-790)\end{array}$ & $870(630-1100)$ & 24 & P3Э* & - & - \\
\hline 12 & $\mathrm{Ca}$ & $\begin{array}{c}11000 \\
(8300-19000)\end{array}$ & $\begin{array}{c}21000(11000- \\
25000)\end{array}$ & 25 & Th & - & - \\
\hline 13 & $\mathrm{Ti}$ & $61(54-72)$ & $48(21-110)$ & 26 & $\mathrm{U}$ & - & - \\
\hline
\end{tabular}

* Р3Э - редкоземельные элементы

анализ методом масс-спектрометрии. Результаты определения элементов в исходных пробах буровых растворов методом масс-спектрометрии даны в табл. 2.

Как видно из представленных результатов, все пробы содержат значительные количества $\mathrm{C}, \mathrm{K}, \mathrm{Na}, \mathrm{Ca}$ и др. На фоне этих элементов содержание урана и тория определить невозможно. Была проведена попытка уменьшить влияние макрокомпонентов разбавлением в 100, 1000 и 10000 раз. Содержание макроэлементов уменьшилось в соответствующее число раз, но и уран, и торий нельзя было определить из-за значительного снижения концентрации в результате разбавления.

Содержание трития определить было невозможно, так как при смешивании с жидким детектором - сцинтилляционным коктейлем - происходило разделение на фазы и эффективность измерения равнялась нулю. Это полностью соответствует полученным ранее данным по воздействию некоторых параметров на гашение люминесценции при определении трития.

Чтобы решить эту проблему, для отделения жидкости, пригодной для определения содержания трития, урана и тория, мы разработали установку для выделения жидкости и соответствующую методику. Основной принцип методики состоит в выделении азеотропной смеси с 
Таблица 3. Содержание трития, урана и тория в анализируемых буровых растворах, среднее значение (диапазон содержания)

\begin{tabular}{|c|c|c|c|c|c|}
\hline \multirow{2}{*}{ Объект } & \multirow{2}{*}{\begin{tabular}{c}
\multirow{2}{*}{ Об Бк/л } \\
\cline { 3 - 6 }
\end{tabular}} & \multirow{2}{*}{ ИСП МС } & \multicolumn{2}{|c|}{$\begin{array}{c}\text {-спектрометрия } \\
\text { мкг/л }\end{array}$} \\
\cline { 3 - 6 } & & ${ }^{238} \mathrm{U}$ & ${ }^{232} \mathrm{Th}$ & ${ }^{238} \mathrm{U}$ & ${ }^{232} \mathrm{Th}$ \\
\hline Район добычи нефти, Сибирь & $7(5-8)$ & $2,9(2.0-3,4)$ & $0,5(0,2-0,7)$ & $3,0 \pm 0,4$ & $0,5 \pm 0,2$ \\
\hline Подземные рассолы, Якутия & $10(8-18)$ & $3,1(2,9-4,0)$ & $0,8(0,4-1,1)$ & $3,4 \pm 0,6$ & $0,6 \pm 0,2$ \\
\hline
\end{tabular}

толуолом, которая ранее использовалась нами при определении содержания трития в различных объектах окружающей среды [21].

В данном случае в отгонной колбе остаются соли, которые не подвергаются возгонке при нагревании в смеси с толуолом при температуре $83{ }^{\circ} \mathrm{C}$. Соотношение пробы к добавляемому толуолу составляло 1:3. Отгонка проводилась досуха, т.е. до полного выделения жидкости из отгонной колбы. Обычно отгонку осуществляли в течение 3 ч. Масса исходного раствора составляла 200 г. Проба взвешивалась непосредственно в отгонной колбе для предотвращения потерь при переносе сосуда, в котором находилась проба при транспортировке, и наличия в том числе следов нефтепродуктов и нерастворимых фрагментов, которые оседают на стенках сосудов.

При определении трития в выделенной жидкости эффективность измерения была 27 \%. Время измерения составляло 720 с.

Результаты определения трития отражены в табл. 3. Минимально детектируемая активность для трития составила 1 Бк/л, которая была определена в соответствии с установленной инструкцией к используемому прибору и с примененнием градуировочных растворов, прилагаемых к прибору.

Уран и торий в полученной жидкости определяли масс-спектрометрическим методом. Для сравнения в табл. 3 приведены результаты определения изотопа урана-238 и тория-232, полученные методом альфа-спектрометрии.

Как видно из представленных результатов, содержание урана-238 и тория-232, определенных двумя методами, хорошо соотносятся друг с другом и различаются только в пределах погрешности. Но предлагаемая подготовка проб отличается существенным сокращением времени подготовки и, следовательно, значительным снижением стоимости самого анализа. Предел обнаружения для урана и тория составил соответственно 20 и 10 нг/л.

Таким образом, предлагаемая методика предварительной подготовки проб растворов со сложной матрицей (буровые, рассолы) отличается высокой эффективностью и экспрессностью. Результаты, полученные для содержания урана, тория при помощи предлагаемого комплекса хорошо сопоставляются с результатами, полученными классическим радиохимическим методом с разделением и электроосаждением на серебряном диске.

Данная методика может быть использована как в лабораторных, так и в полевых условиях. Она требует чрезмерных финансовых вложений, отличается минимальным временем подготовки, что особенно важно при возникновении чрезвычайных ситуаций, когда необходим оперативный анализ для оценки и ликвидации негативных последствий. С успехом может быть 
применена в условиях мегаполисов для скорейшего и наиболее полного мониторинга состояния окружающей среды.

Предложенная методика может оказаться чрезвычайно полезной, на наш взгляд, для определения урана и тория для изучения их миграции на загрязненных радионуклидами территориях, а также в процессах дезактивации редкометального минерального сырья, большая часть которого (монацит, самарскит, браннерит и др.) содержит природные радионуклиды. В свою очередь, разработка и адаптация ключевых технологий переработки РЗЭ продукции выходят на первый план вследствие Постановления Правительства РФ от 15 апреля 2014 г. «Об утверждении государственной программы Российской Федерации «Развитие промышленности и повышение её конкурентоспособности».

\section{Список литературы}

1. American Petroleum Institute, Bulletin on management of naturally occurring radioactive materials (NORM) in oil \& gas production: American Petroleum Institute. Washington, D.C., API Bulletin E2, 1992. 45 p.

2. Baskaran M. Handbook of Environmental Isotope Geochemistry / M. Baskaran. V. 1, Springer, Verlag, Berlin, Neidelberg, 2011. 950 p.

3. Otto G.H. A national survey of naturally occurring radioactive materials (NORM) in petroleum producing and gas processing facilities / G.H. Otto. American Petroleum Institute, Dallas, Texas, 1989. $265 \mathrm{p}$.

4. Бондарева Л.Г., Болсуновский А.Я., Трапезников А.В., Дегерменджи А.Г. Использование новой методики концентрирования трансурановых элементов в пробах воды р. Енисей. Доклады академии наук, 2008. 423 (4). C. 479-482. [Bondareva L.G., Bolsunovsky A.Y., Trapeznikov A.V., Degermendzhy A.G. Using a new technique of concentrating of transuranic elements in water samples p. Yenisei. Reports of the Academy of Sciences, 2008. 423 (4), P. 479-482. (In Russ.)]

5. Fisher R.S. Geologic and Geochemical Controls on Naturally Occurring Radioactive Materials (NORM) in Produced Water from Oil, Gas, and Geothermal Operations. Environmental Geosciences. 1998. (5). P. 139-150.

6. Gilkeson R.H. Isotopic and geologic studies to identify the sources of sulfate in groundwater containing high barium concentrations / R.H. Gilkeson, E.C. Perry Jr., K. Cartwright. Water Resources Center, Report 83-0165, Urbana-Champaign, IL., 1981. 39 p.

7. Silve N.C., Fernandes E., Cipriani M., Taddel M. The natural radioactivity ofBrazilian phosphogypsum. Journal of Radioanalytical and Nuclear Chemistry, 2001. 249 (1), P. 251-255

8. Wang J.J., Chen I.J., Chiu J.H. Sequential isotopic determination of plutonium, thorium, americium, strontium and uranium in environmental and bioassay samples. Appl.Radiat.Isot., 2004. 61(2-3). P. 299-305.

9. Минимальная измеряемая активность. Понятие и использование в радиометрии / Е.И. Григорьев [и др.]. Аппаратура и новости радиационных измерений. 1995. (4). С. 52-58. [The minimum measurable activity. The concept and use of radiometry / EI Grigoriev [et al.]. The equipment and the news of radiation measurements.1995. (4). C. 52-58. (In Russ.)]

10. Lochamy J.C. The Minimum Detectable Activity Concept. National Bureau of Standards, SP456. 1976. Pp. 169-172. 
11. International Atomic Energy Agency.Modeling the environmental transport of tritium in the vicinity of long-term atmospheric and sub-surface sources, IAEABIOMASS-3, IAEA, Vienna, 2003.

12. Davis P.A., Kotzer T.G., Workman W.J.G.Environmental tritium concentrations due to continuous atmospheric sources. Fusion Science and Technology, 2002.(41). 453-457.

13. Horwitz E.P., et al. Separation and Preconcentration of Actinides from Acidic Media by Extraction Chromatography. Analytica Chimica Acta, 1993. 281, P. 361-372.

14. International Atomic Energy Agency. Handbook of Parameter Values for the Prediction of Radionuclide Transfer in Terrestrial and Freshwater Environments. IAEA-TRS-472, IAEA: Vienna, 2010.

15. International Atomic Energy Agency, Fundamental safety principles: safety fundamentals. IAEA safety standards series No. SF-1, IAEA, Vienna, 2006.

16. International Commission on Radiation Protection, Recommendations of the International Commission on Radiological Protection Publication 103 Annals of the ICRP 37 2-3, 2007.

17. Fukutani S., Fukui M., Akio Koyama A. and Nishimaki K. A Method of Calibration for Measurement of Low Level Tritium in Environmental Water using a Liquid Scintillation Counter. Journal of Nuclear Science and Technology. 2008. (6), P. 89-92

18. Wood M.J., McElroy R.G., Surette R.A., Brown R.M. Tritium sampling and measurement. Health Phys. 1993. 65, (6). P. 610-627.

19. Gusev N.N., Dmitriev P.D. Radioactive chain. Handbook. Energoatomizdat, 1988.

20. Дерффель К. Статистика в аналитической химии. М.: Мир, 1994. 267c. [K.Derffel Statistics in Analytical Chemistry. M .: Mir, 1994. 267 p. (In Russ.)]

21. Bondareva L. Natural Occurrence of Tritium in the Ecosystem of the Yenisei River. Fusion Science and Technology. 2011. 60 (4), P. 1304-1307. 